\title{
Using the Web to Connect Research and Practice: Towards a Discipline of Evidence-Based Practice
}

\author{
Daniel L. Moody \\ Norwegian University of Science and \\ Technology (NTNU) \\ Trondheim, Norway \\ dmoody@idintnuno \\ School of Business Systems, \\ Monash University \\ Melbourne, Australia 3800 \\ dmoody@infotech.monash_edu_aw
}

\begin{abstract}
In most disciplines, research findings take a long time to filter into practice, if they ever do at all. The result of this is under-utilisation of research results and sub-optimal practices. There are a number of reasons for the lack of knowledge transfer. Firstly, practitioners don't have the time to keep up with the latest research - in professional practice, there is little time available to read academic journals. Once they have completed their formal education, they tend to rely on tacit knowledge acquired through experience rather than explicit knowledge in the form of academic research. In addition, the volume and geometric growth of research literature results in a massive problem of information overload-it is not humanly possible for practitioners (and challenging even for full-time researchers) to keep up with all the research published in their field. From the "supply side", academic research is primarily focused on the production rather than distribution of knowledge-there is little investment in the distribution of research results beyond academic communities. The Internet provides a potential solution to this problem, in that it provides a common information infrastructure for connecting those who produce knowledge (researchers) and those who need to apply it (practitioners). This paper describes two projects which use the Internet to make research results directly available to practitioners in the workplace. The first is a project in a health department which provides medical staff with on-line access to the latest medical research at the point of care. The second is a project currently in progress to implement a similar system to support IS practitioners. Finally, we draw some general lessons about how to improve transfers of knowledge from research and practice, which could be applied in any discipline.
\end{abstract}

Keywords: knowledge management, evidence based medicine (EBM), Internet, World Wide Web, webbased development, IS research, IS practice, education, decision support

\section{Introduction}

\section{Transfer of Knowledge Between Research and Practice}

Material published as part of these proceedings, either on-line or in print, is copyrighted by Informing Science. Permission to make digital or paper copy of part or all of these works for personal or classroom use is granted without fee provided that the copies are not made or distributed for profit or commercial advantage AND that copies 1) bear this notice in full and 2) give the full citation on the first page. It is permissible to abstract these works so long as credit is given. To copy in all other cases or to republish or to post on a server or to redistribute to lists requires specific permission from the publisher at Publisher@Intormingscience.org
There is an enormous amount of new knowledge generated every year as a result of academic research. To make a practical difference, this knowledge needs to be disseminated and used in practice. Knowledge has no real value on its own-it only becomes valuable when people use it (Sveiby, 1997). However in most disciplines, research findings take a long time to filter into practice, if they 
ever do at all. There are a number of barriers to the flow of knowledge between research and practice, which originate from both sides of the divide:

- $\quad$ Researcher's viewpoint (supply side): Academic research is primarily focused on production rather than distribution of knowledge (Gibbons et al, 1994). Research communities have developed highly efficient mechanisms for transfer of knowledge among themselves (via the processes of publication and citation), but there is little investment in the dissemination of research results beyond this. Universities reward researchers for publication in scholarly journals and conferences rather than whether their ideas are applied in practice. In manufacturing, it is important to pay equal attention to production and distribution. To get maximum value from investments in production of goods, it is necessary to have parallel investments in distribution, to make sure goods get sold and produce revenue. Similarly in academic research, to get maximum value from investments in research, it is necessary to invest in dissemination and application of research results in practice and achieve social outcomes.

- $\quad$ Practitioner's viewpoint (demand side): The volume of research published every year means that practitioners could not possibly keep up with all the latest research in their field-if they did, they would have little time to do anything else. Also, the pressures of professional practice leaves little time to read academic journals: time is money, and most commercial organisations do not reward their employees for keeping up with research. Also, practitioners often have difficulty understanding academic papers, which are mainly written for an audience of other researchers (members of editorial boards and review committees).

The issue of how to transfer research results into practice is rarely addressed in published research, and requires much more than publication in scholarly journals, which is normally seen as the endpoint of a research project) (Gibbons et al, 1994).

\section{Current Knowledge Distribution Model}

Until now, there has been an implicit assumption that the best way to transmit research knowledge into practice is to first load it into human minds, via the long and expensive education of professionals. However there are enormous "voltage drops" along this transmission line (Weed, 1997):

- Only a portion of research knowledge can be loaded, given the limitations of human information processing and the limited time available for their education.

- Only a portion of the knowledge loaded is retained, and in fact most is quickly forgotten after the examination.

- Only a portion of the knowledge loaded is ever used. Because it is difficult to predict what knowledge will be useful in the future, as much information as possible is loaded in order to allow for all contingencies. This results in information overload and further taxing of the retention powers of the human mind.

- Much of the knowledge that is retained quickly becomes obsolete, and there is no assurance that it will be replaced by relevant new knowledge.

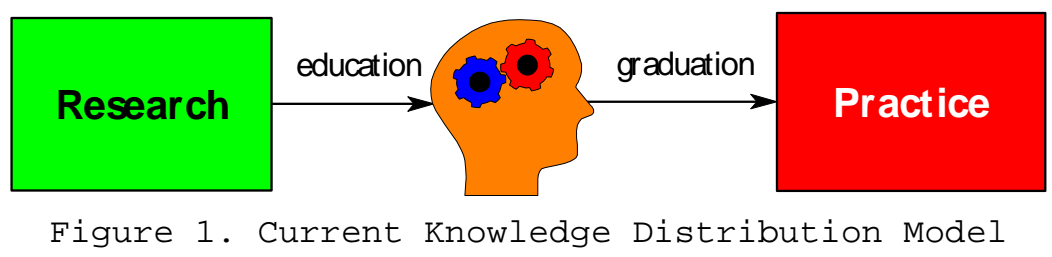

In the "Information Age", this seems a rather archaic and inefficient way of transferring knowledge. Just as science developed the microscope to magnify the power of the human eye, there is a similar need to use 
information technology to expand the human mind's limited capacity to store and recall large amounts of information. In an ideal world, practitioners should not need to load enormous amounts of information and then try to retrieve it years later when it has become outdated, but should have the latest research knowledge at their fingertips in the workplace. This will not substitute for the education process, but will provide a way of continuously and selectively updating it - a process of "just-in-time" learning. The education process provides only a baseline of understanding: a "snapshot" of knowledge in the field at a point in time. Whenever a practitioner encounters a new type of problem, they should be able to update their knowledge on an "as needed" basis. Rather than loading every piece of information that might be useful, only the essentials need to be loaded initially, and this is continuously supplemented by problem-directed knowledge acquisition.

\section{Knowledge Management}

Fundamental to the problem of linking research and practice is the concept of knowledge management. Knowledge management has only recently emerged as a discipline in its own right (Sveiby, 1997; Davenport and Prusak, 1998). Knowledge is a high value form of information that can be used to make decisions and take action (Davenport et al, 1998). A key difference between knowledge and information or data is that it is intellectually intensive rather than IT-intensive-knowledge is the result of human interpretation and analysis rather than data processing. Knowledge can be classified as either:

- $\quad$ Tacit: knowledge stored in people's heads

- Explicit: knowledge which has been written down or codified

Formal education focuses primarily on imparting explicit, factual knowledge ("knowledge that"), while experience in practice supplements this with tacit, procedural knowledge ("knowledge how") (Sveiby, 1997). Managing knowledge of both kinds has become a major concern in many organisations and is increasingly being seen as a source of sustainable competitive advantage (Nonaka and Takeuchi, 1995;

Davenport et al, 1998; Hansen et al, 1999). So far, knowledge management has been primarily concerned with the creation and dissemination of knowledge within a single organisation. However the same principles can be applied to the issue of managing knowledge transfers between research and practice.

There are two key flows of knowledge which need to occur between research and practice (Figure 2):

- $\quad$ Practice $\rightarrow$ Research: research activity should be driven by the needs of practice. This ensures that research is relevant.

- $\quad$ Research $\rightarrow$ Practice: research results should be disseminated and applied in practice. This ensures that research actually leads to improvements in practice (impact).

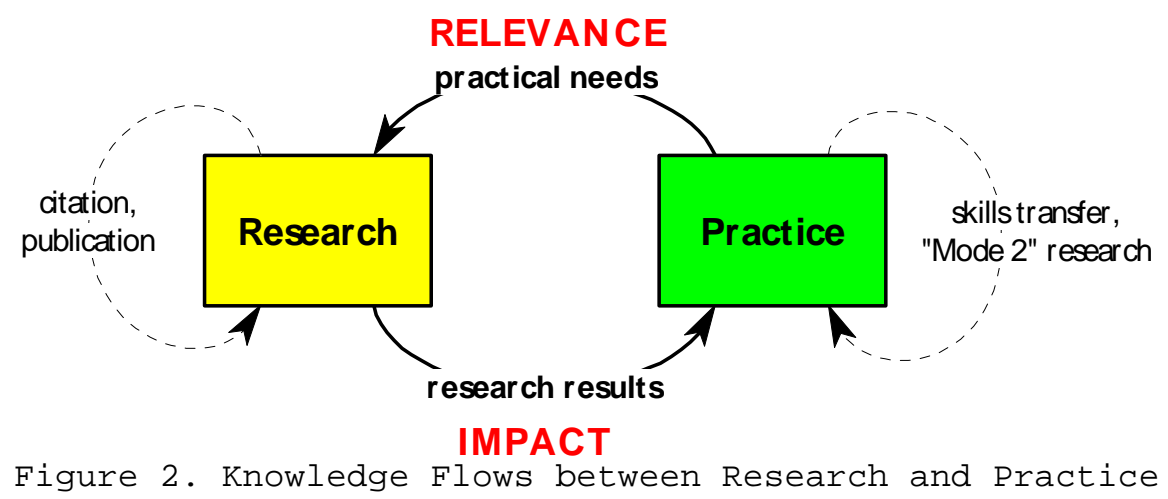

There are also flows of knowledge within each community, although these are not the concern of this paper: 
Using the Web to Connect Research and Practice

- $\quad$ The processes of citation and publication are used to transfer knowledge among researchers.

- The process of skills transfer, in which tacit knowledge is transferred via on-the-job learning in the workplace.

- Industry-based research and development, or "Mode 2" research, in which knowledge is generated in the context of a practical problem rather than from within a disciplinary framework (Gibbons et al, 1994).

Building better connections between research and practice will result in more effective production and application of research knowledge. In this paper, we focus on the flow of knowledge from research to practice (impact), but this will also improve the relevance of research via natural feedback processes. Improving the flow of knowledge in one direction should also improve the flow of knowledge in the reverse direction.

\section{Objectives of this Paper}

Improving connections between research and practice requires a channel which allows the transfer of both explicit and tacit knowledge. We argue that the World Wide Web provides a global infrastructure for connecting researchers and practitioners. This paper describes two projects which use the Internet to make research knowledge directly available to practitioners in the workplace. The first is a project in a health department which provides medical staff with on-line access to the latest medical research at the point of care, in order to improve the quality of clinical decision making. The second is a project currently in progress to implement a similar system to support IS decision making. Finally, we draw some general lessons from these projects about transferring knowledge from research and practice.

\section{Case Study 1: Evidence Based Medicine}

\section{Medical Decision Making}

To make appropriate decisions about patient care, medical practitioners need to take into account all relevant medical knowledge and integrate it with detailed data about the patient's condition. Access to the latest medical research can mean the difference between life and death, an accurate or erroneous diagnosis, early intervention or a prolonged and costly stay in hospital (Ayres and Clinton, 1997). However staying abreast of all the latest developments in medical research is a daunting task - it is estimated that the amount of medical knowledge doubles every five years (Weed, 1997). Faced with information overload, doctors fall back on global judgements based on experience rather than thorough analysis of the relevant medical literature. As a result, medical practice is surprisingly anecdotal rather than being based on scientific fact.

For decades, the medical profession has documented high levels of error, far higher than most other industries would tolerate. An adverse event is defined as "an unintended injury or complication which results in disability, death or extended hospital stay, and is caused by health care management rather than the patient's disease" (Wilson et al, 1995). This corresponds roughly to an "error" in medical practice. It is estimated that 180,000 people die each year in the United States as a result of adverse events- the equivalent of three jumbo jet crashes every day (Leape, 1994). A study of the Australian health system found that $16.6 \%$ of public hospital admissions resulted in adverse events (Wilson et al, 1995). When the causes of adverse events are investigated, it is found that most (over 80\%) are the result of decision making errors (Wilson et al, 1999).

One of the reasons for the high levels of error in medical practice is its reliance on the unaided human mind (Weed, 1997). Psychological research shows that the human mind is strictly limited in how much 
information it can memorise and recall (Miller, 1956; Baddeley, 1994; 1999; Eysenck and Keane, 2000). In normal medical practice, doctors rely almost exclusively on tacit knowledge accumulated as a result of their medical training and subsequent clinical experience. As a result, decisions about the most appropriate treatment for patients are often made based on knowledge which is out-of-date, incomplete and anecdotal. As Weed (1997) says:

"We should never have placed so much power in the hands of those who memorise knowledge, regurgitate it in examinations, forget much of it, specialise in a small piece of it, and never fully integrate what they do with the details of patient's needs."

\section{Evidence Based Medicine}

Medical research findings take a long time to filter into clinical practice (Phillips, 1998). Empirical studies have shown that on average, there is an 8-13 year time lag (depending on the specialty) between a treatment being proven to work and its adoption in common practice. It has also been found that $70 \%$ of treatments currently in use do not have sufficient evidence to support that they are any more effective than doing nothing (Chalmers, 1993). One of the major barriers to the implementation of research findings is the volume and geometric growth of the medical literature. It is not humanly possible to keep up with all the advances in all areas of medical research - it is estimated that the average medical practitioner would have to read 20 articles per day just to keep up with the latest developments in their specialty area. (Jordens et al, 1998). It is also difficult for clinicians to make sense of the often-conflicting research findings in a particular area. Medicine has well-developed mechanisms for transferring tacit knowledge, via the internship process (on the job learning). However for transfer of explicit knowledge, it relies mainly on the formal education process. There is surprisingly little use of information technology in clinical practice. Medical practitioners use IT to support administrative tasks (billing, practice administration) but less in diagnosis and treatment. A survey of general practitioners in Australia in 1998 showed that less than $10 \%$ of GPs used computers for clinical applications.

Recognition of such problems led to the discipline of evidence based medicine (EBM). Evidence based medicine is a discipline which synthesises research findings on the effectiveness of medical treatments to support clinical decision making (Sackett et al, 1997). The purpose of EBM is to ensure that decisions about patient care to be based on the latest scientific evidence, where "evidence" is generally restricted to the results of randomised clinical trials (RCT). The aim is to reduce the time lag between the development of new treatments and their use in everyday medical practice, and to avoid the use of unproven or disproven treatments. The principles of EBM can be summarised as follows:

- $\quad$ To use treatments that work

- To eliminate treatments that don't work

- To conduct research into treatments whose effects are unknown

One of the major methodological tools in EBM is the systematic review (Cochrane, 1972). Systematic reviews begin with an exhaustive search for published and unpublished research studies addressing a particular clinical issue (e.g. treatment of asthma). The next step is to critically evaluate the studies to identify which are of sufficient quality to contribute to decision making. The final step is to pool the results of the studies to arrive at a quantitative estimate of the effectiveness of the treatment(s). Reviews must also be regularly updated to take account of new research developments.

Synthesising the research evidence is only the starting point for using research to improve practice. Equally important is the dissemination and use of this information. To make a practical difference, systematic reviews must be readily available to medical practitioners, and must be actively used in everyday clinical practice. EBM represents an application of knowledge management in the medical field, although it pre-dates the mainstream knowledge management literature by more than two decades. It focuses on 
synthesising explicit knowledge in the form of research findings, and using this knowledge in clinical decision making.

\section{Organisational Context}

This case study took place in one of Australia's state health departments. The department is one of the largest organisations in Australia, with a budget of over six billion dollars in 1998 and over one hundred thousand staff. Historically, clinical information needs had not been well supported by investments in information technology. The majority of information systems in the organisation supported administrative processes (e.g. financial systems, payroll systems, patient administration systems), with very few systems directly supporting patient care. To address this issue, a committee was formed in September 1995, to specify requirements for clinical information at the point of care. It consisted of fifty clinicians from all health disciplines including hospitals, general practice, community health and universities. A proposal was developed for a web-based system called the Clinical Information Access Project (CIAP) to meet this need, which was endorsed by senior management in December 1996. The stated objective of the project was:

"To provide clinicians with access to on-line medical information to support clinical practice, education and research at the point of care".

A pre-implementation survey of 2757 clinicians and medical librarians was carried out to identify the most important knowledge sources to support clinical practice. The system went live on July 4, 1997, taking just over six months from its initial inception to implementation. It has now been in operation for over four years, and is still evolving.

\section{Knowledge Content}

The knowledge content of the system was based on the requirements identified in the pre-implementation survey. It consists of five major components, which correspond to different classifications of knowledge on the tacit/explicit dimension and the internal/external dimension (whether the knowledge was produced inside or outside the organisation), as shown in Table 1. Unlike most knowledge management systems, which primarily focus on internal knowledge (Sveiby, 1997; Davenport et al, 1998; Davenport and Prusak, 1998; Hansen et al, 1999), this system is mainly focused on providing access to external knowledge, and in particular, medical research knowledge.

Table 1. Knowledge Components

\begin{tabular}{|l|l|c|}
\cline { 2 - 4 } & \multicolumn{1}{c|}{ EXTERNAL } & INTERNAL \\
\hline \multirow{2}{*}{ EXPLICIT } & Systematic (EBM) Reviews & \\
& Pharmaceutical Databases & Clinical Policies and Protocols \\
\hline TACIT & & Listservers \\
\hline
\end{tabular}




\section{Systematic Reviews}

A range of databases are provided which provide on-line access to systematic (EBM) reviews:

- Cochrane Library: this is an electronic database of systematic reviews produced by the Cochrane Collaboration, which is an international not-for-profit organisation specialising in principles of EBM. The Cochrane Library is recognised as the leading source of EBM reviews.

- $\quad$ APC EBM Reviews: systematic reviews produced by the American College of Physicians.

- Evidence-Based Medicine: systematic reviews produced by the British Medical Association.

- Database of Abstracts of Reviews of Effectiveness: systematic reviews produced by the British National Health Services' Centre for Reviews and Dissemination (NHS CRD).

These databases provide very high value knowledge (external explicit knowledge) in the form of synthesised research findings. Each EBM review incorporates thousands of hours of critical review and synthesis of research articles by the leading researchers and practitioners in the world.

\section{Literature Searching Tools}

Access to primary research sources (medical research journals) is also provided, as systematic reviews are only available for a relatively small percentage of medical conditions:

- MEDLINE: this is produced by the U.S. National Library of Medicine, and is recognised as the leading bibliographic source of medical research. MEDLINE provides on-line searching with links to full text medical journals.

- $\quad$ CINAHL: provides on-line searching with links to full text nursing and allied health journals.

- $\quad$ PsychINFO: provides on-line searching with links to full text psychiatry and psychology journals

- Healthstar: provides on-line searching with links to full text health administration journals

- Full text journals and textbooks: a range of full text journals and medical textbooks are available online.

These databases provide access to the latest medical research findings. Compared to EBM reviews, they have the disadvantage that clinicians must synthesise the research themselves, but have the advantage of broader coverage (EBM reviews are only available for a limited range of topics) and currency (EBM reviews are only updated on an annual basis).

\section{Pharmaceutical Databases}

With thousands of drugs currently on the market, and hundreds of new ones released every year, it is impossible for clinicians to keep information about all available drugs in their minds (Milne, 2002). The system provides a range of sources of drug information:

- MIMS: a comprehensive pharmaceutical database, which includes details of all known drugs, sideeffects, interactions and recommended dosages.

- Antibiotic Guidelines: provides decision support in prescribing antibiotics (which are generally poorly prescribed in practice).

- Micromedex: poisons and toxins information.

Up-to-date drug information (e.g. recommended dosage, administration instructions, allergies, side-effects and interactions) is essential for prevention of adverse events. A study of adverse events in general practice found that over 50\% of adverse events were the result of pharmacological errors (Kidd and Veale, 1998). Also, over $10 \%$ of adverse events in Australian public hospitals are caused by inappropriate use of drugs (Wilson et al, 1999). 


\section{Clinical Policies and Protocols}

Clinical policies and protocols define standard procedures for handling particular types of cases (e.g. cardiac arrest, road trauma), and play a critical role in medical practice. Incorrect protocols or failure to follow protocols represent the most common cause of adverse events in clinical practice (Wilson et al, 1995; Wilson et al, 1999). They represent an important source of explicit knowledge in the organisation. Each hospital defines its own policies and protocols, and there may be wide variations between them based on equipment available and when they were last updated. The system allows clinicians to post their clinical policies and protocols on a voluntary basis for peer review. The purpose of this is to encourage collaboration and sharing of knowledge, and to move towards standardisation and development of "best practice" policies and protocols.

\section{Listservers}

Listservers are provided for on-line discussion of problems and issues in particular areas of specialty, for example, asthma, stroke and medical ethics. This facilitates the exchange of ideas and experiences among researchers and clinicians across the state. This is especially useful for clinicians in rural and remote areas, who have less opportunity for face-to-face exchange of knowledge. Listservers facilitate the transfer of tacit knowledge between individuals (both clinicians and researchers).

\section{Scenario_How the System is Used}

To illustrate how the system is used in everyday clinical practice, consider the case of a patient who presents at a hospital with funnel web spider bite. The funnel web spider (Atrax Robustus) is unquestionably the most dangerous spider in Australia. It is a large $(6-7 \mathrm{~cm})$, black, aggressive spider, with fangs large and powerful enough to penetrate a fingernail. During a bite the spider firmly grips its victim and bites repeatedly, and the venom is highly toxic. There is estimated to be 30-40 cases of funnel web spider bite occur each year in Australia. Given the rarity of such cases, it is unlikely that a

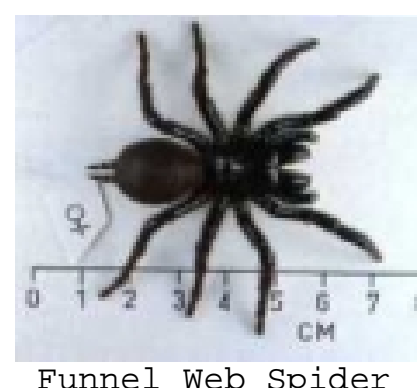

Funnel Web Spider clinician would be able to rely on previous experience or their medical training to know how to treat such a case. Death occurs between 15 minutes and 3 days following the bite, so prompt action is essential.

From the opening screen, the attending medical officer conducts a search using the phrase "funnel web spider bite". This initiates a search across all information sources in the system. 


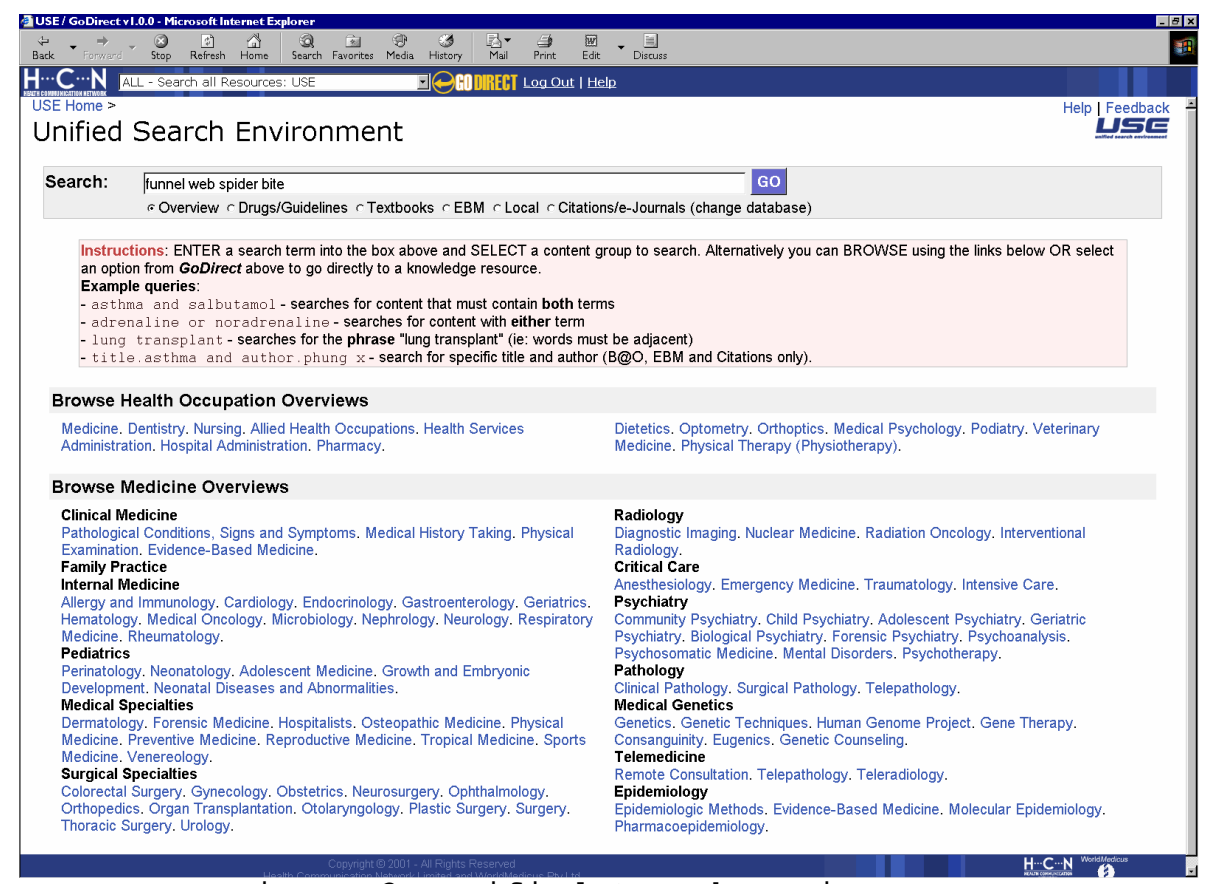

Figure 3. Unified Search Environment

The top-ranked result of the search is an entry from the Drug and Therapeutic guidelines, which describes the symptoms of funnel web spider bite (for confirming the diagnosis), the antivenom required, recommended dosage, administration instructions, interactions and possible side effects (for treatment).

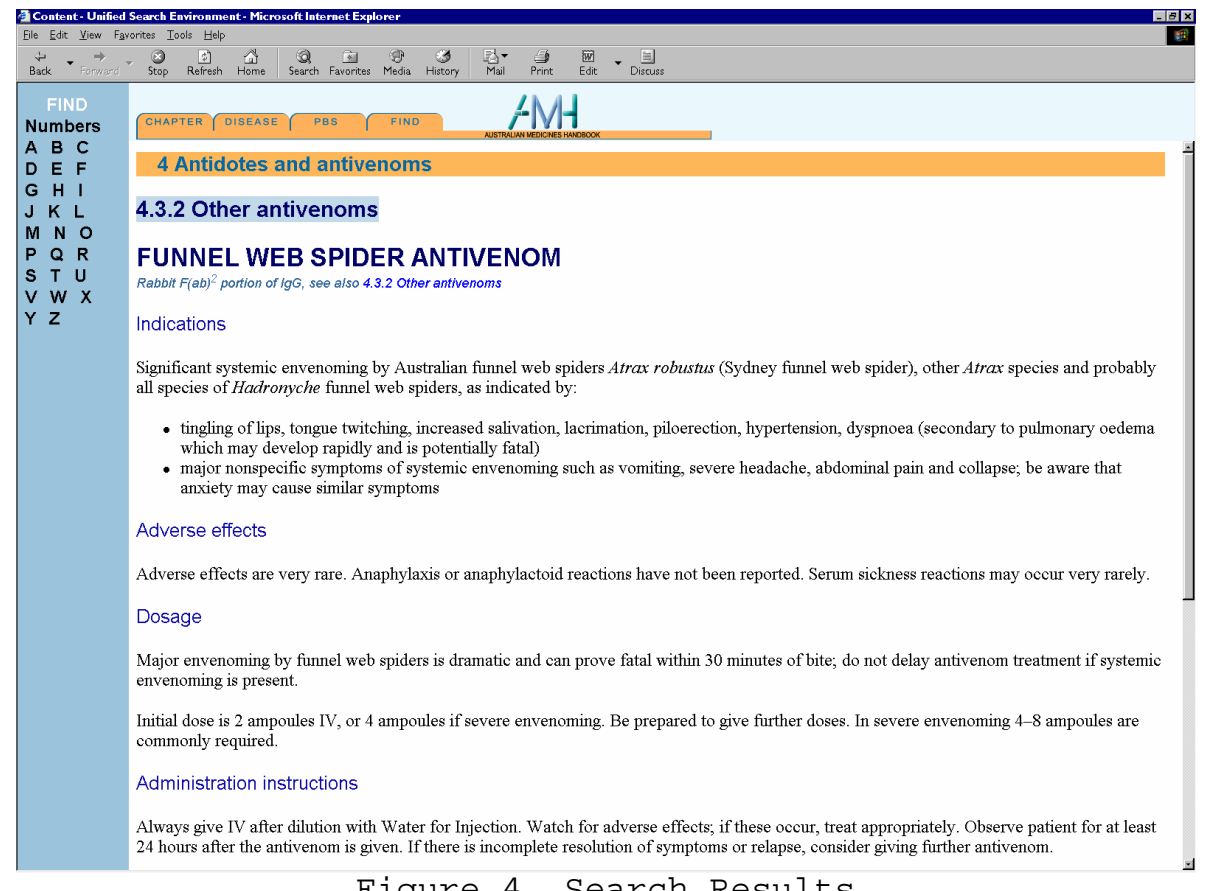

\section{Technology Architecture}

The system operates using a single web server located at head office. The Internet was chosen instead of intranet technology in order to maximise the "reach" of the system. Because of the geographic spread of the organisation and the lack of a communications network linking all health care facilities, an intranet would have excluded a large proportion of clinicians. Using the World Wide Web, all that is required to 
Using the Web to Connect Research and Practice

use the system is a PC, a modem and Internet access. An added advantage of using the Internet is that it provides the flexibility for clinicians to access the system from home. The system is available 24 hours a day, 7 days a week.

\section{Organisational Impact}

Both anecdotal evidence and perceptions of clinicians support the conclusion that the system has been successful in improving the quality of clinical decision making. An on-line survey of clinicians using the system revealed that $90.82 \%$ felt that it had improved patient care. There have also been a number of reported cases where access to the system has saved lives:

- A clinician in a rural hospital was able to save a patient in a critical condition suffering from the Lyssavirus. The Lyssavirus, which is acquired from contact with bats, causes encephalitis in humans and can be fatal if not treated quickly. However because it is a relatively new disease (the first case was discovered in Australia in May 1996), it does not yet appear in medical textbooks. Using MEDLINE, the clinician was able to look up and apply the appropriate treatment.

- Another rural clinician was able to save a patient who was admitted in a critical condition in the middle of the night, suffering from meningitis. The patient did not respond to the normal treatment for this condition, indicated by the standard clinical protocol. A MEDLINE search revealed a new drug treatment which had immediate results.

- Finally, a clinician on the north coast was able to save a child who had been bitten by a funnel web spider by looking up the anti-venom in the poisons database in Micromedex. For the anti-venom to be effective, it must be applied within 30 minutes, so having on-line access to this information meant the difference between life and death.

\section{Postscript: A Revolution in Health Care?}

This system has the potential to revolutionise the practice of health care. It provides a mechanism for moving away from experience/anecdotal/memory based medical practice and towards evidence based practice. It also provides a channel for transfer of research results to clinical practice. One of the explanations for the success of this system is that unlike traditional DSS (which have proven to be largely unsuccessful in medical practice) this system does not tell the clinician what decision to make. Twenty years of DSS research has revealed a persistent problem, in that decision makers either ignore or modify the advice given by the DSS, even when shown how good it is and that they are unlikely to improve on it (Lim and O'Connor, 1995; Turban, 1995; Lawrence and Sim, 1999; Goodwin, 2000). Instead of accepting the system's advice, decision makers tend to trust their own judgement much more. A possible explanation for this is that human nature being what it is, people, and especially highly educated and experienced professionals, do not like being told by a computer what to do. This system supports the first stage of decision making (the intelligence phase), by providing information about available treatments and the evidence for and against them. However it empowers human judgement in the later decision making phases (design and choice), where no computer can substitute for experience and expert judgement (and where no expert would be likely to accept its recommendation anyway!).

If use of the system was extended to consumers, it would have an even greater impact. For example, patients could use the system to look up the latest medical research about their treatment. If they found that (a) there was no research evidence to support use of the treatment they were being given, or (b) there was a better, clinically proven treatment available, they would rightly question their doctor's decision. The medical profession currently enjoys almost "god like" status in the community, largely as a result of the wide knowledge gulf between medical practitioners and consumers. Patients are often uninformed, passive objects in the medical care process, and rarely question their doctor's advice. Making the latest re- 
search evidence available to consumers would remove the knowledge gulf between doctors and patients, and would help to improve accountability and the quality of health care.

\section{Case Study 2: Evidence Based IS Practice}

\section{Information Systems Research}

Information Systems (IS) is the study of phenomena associated with planning, developing, implementing, maintaining, using and managing information systems. The goal is to better understand these phenomena so that individuals, groups, organisations and society in general can use information systems more effectively and more efficiently (Weber, 1997). Like medicine, it is an applied rather than a pure discipline (Moody, 2000).

The IS profession exhibits far more serious problems than the medical profession in terms of its links with research. In medicine, the accreditation process requires that medical practitioners have formal qualifications, regularly update their qualifications, subscribe to medical journals and attend conferences in order to practice. The fact that demand for IT skills far exceeds supply makes it difficult to impose accreditation requirements as in more established professions such as accounting, law or medicine. This means that people can practice in the IS industry without relevant qualifications or experience, and have no requirements to stay up to date with the latest research. This leads to serious problems with the status of IT as a profession, and the use of questionable and outdated practices.

There is a major "disconnect" between research and practice in the IS field (Figure 5). Knowledge flows take place almost entirely within each community:

- Research results are disseminated among research communities via publication in journals and conferences but rarely find their way into practice-IS practitioners rarely read academic journals.

- Practitioners develop solutions to practical problems without referring to academic research or publishing the results in academic journals and conferences.

IS practitioners rarely refer to research evidence to make important decisions but instead rely on their experience, talking to peers, articles in the popular press or advice from consultants and vendors. This means that, like medical practitioners, but to a far greater extent, they are operating from an incomplete and biased knowledge base.

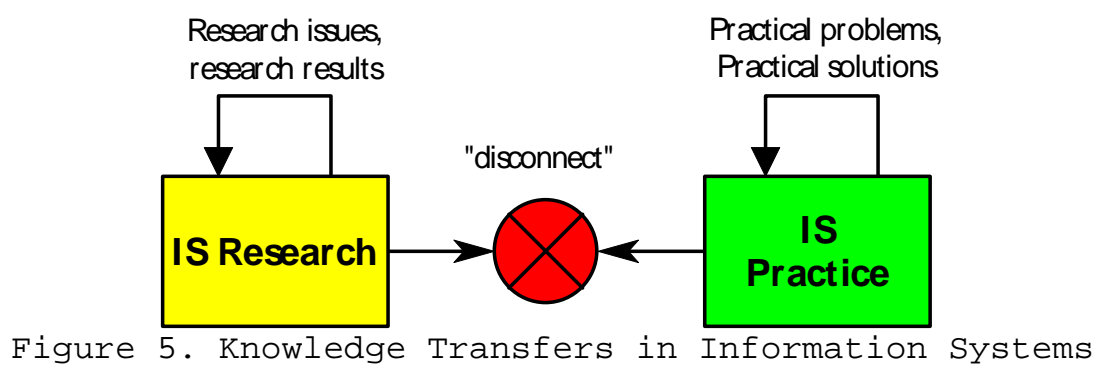

Like medical practice, IS practice exhibits high levels of errors. For example, only $16.2 \%$ for software projects are completed on-time and on-budget (Standish Group, 1995) - this represents a success rate about the same as the error rate in medical practice! Part of the reason for the error rates in IS practice is the failure to learn from mistakes and the persistent use of practices which do not work. Researchers play a vital role in enabling a profession to learn from its mistakes and by collecting evidence about the effectiveness of practices. However rather than consult published research, practitioners tend to rely instead on experience (their own and their peers) or "expert opinion" (from vendors, consultants and the popular 
press). However this knowledge is largely anecdotal and highly subjective. A number of industry research organisations have emerged to address this need. However while this research is more accessible to the average practitioner and relevant to everyday practice than academic research, it is of questionable validity.

\section{Applying the Principles of EBM to IS Practice}

In this case study, we look at how the knowledge management approach used in the first case study can be applied in the IS profession. This is an example of analogical reasoning, a problem solving approach in which a solution is found to a problem by looking at how a similar problem has been successfully solved in another (referent) domain. The solution to this second problem, the exemplar, can be used as a basis for developing a solution to the original (or target) problem (see Figure 6). In this case:

- The target domain is IS, while the referent domain is medicine;

- The problem to be solved is that of transferring IS research knowledge to IS practice, while the exemplar problem is the transfer of medical research knowledge to clinical practice;

- The exemplar solution is the web-based system to support evidence based medical practice described in the first case study, while the target solution is a system to support evidence based IS practice.

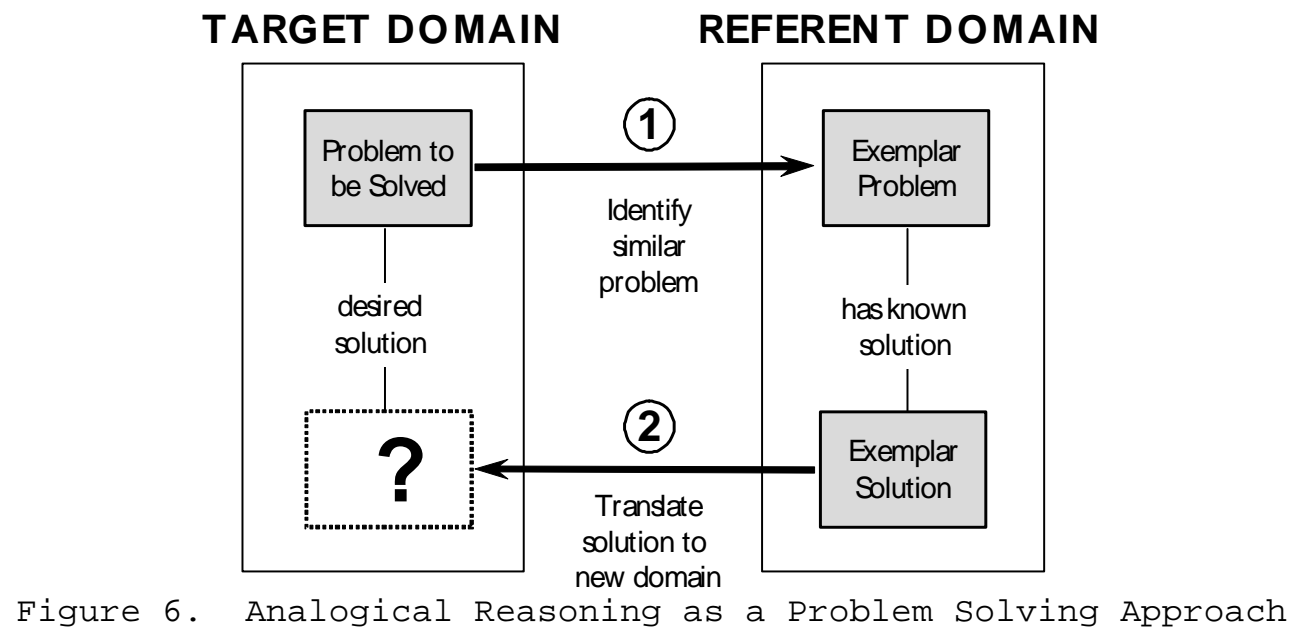

The IS profession is very immature. It has only existed since the early 1960's, while other professions, like medicine, law and engineering, have roots back to antiquity. Analogical reasoning represents a particularly useful research approach for a relatively young discipline like information systems, and a way of learning from other, more established disciplines. There are also important similarities between IS and medicine. Both are applied disciplines, which focus on applying technology to solve practical problems. While medical practitioners apply medical interventions to improve the health of their patients, IS practitioners apply IT-based interventions to improve personal and organisational effectiveness.

\section{Improving the Impact of IS Research: Towards Evidence Based IS Practice?}

There is an enormous amount of IS research published each year. However very little of this knowledge finds its way into IS practice. One reason for this is the huge volume of research papers published every year. A recent study identified over 233 journals in the MIS field (Hardgrave and Walstrom, 1997). This is partly the result of the "publish or perish" phenomenon, which results in the creation of more and more publication outlets to provide opportunities for academic promotion (Weber, 1997). The result is a situa- 
tion of massive information overload for IS practitioners. The average practitioner would not have time to read all the articles published in their area of interest, even if they spent all their time doing so.

The idea behind EBM - of making decisions based on research evidence rather than experience, opinion or anecdotal evidence-is something which is badly needed in IS. This would help to improve the uptake of research ideas in practice, reduce the time lag between research innovations and their implementation in practice, provide a basis for learning from mistakes and reduce the use of suboptimal practices. The objectives of evidence based IS practice would be to:

- Use practices that have been proven to work

- Eliminate practices that do not work

- Conduct research into practices whose effectiveness is unknown

The latter would provide clear direction for IS research, which would improve its relevance to practice (the feedback loop in Figure 2). The concept of systematic reviews, of synthesising research results to support decision making, provides a way of making research results more accessible to practitioners, and increases the likelihood that they will be used in practice (Moody, 2000).

\section{Project Objectives}

The objective of this project is to develop a web-based system to provide access to the latest research to IS practitioners in the workplace. This project is a joint venture between Monash University and the Australian Computer Society (ACS). The ACS is the professional association for IS practitioners in Australia. The design of the system will be closely based on the CIAP system described in the first case study. The system will incorporate:

- On-line literature searching: this would provide sophisticated searching of IS journals with links to full text articles. This facilitates the transfer of explicit knowledge between research and practice. A number of existing on-line literature search engines could be used for this purpose. Such tools are currently almost exclusively the domain of researchers, but would also be useful in practice.

- Systematic reviews: this would involve the formation of expert committees, consisting of both researchers and practitioners, to review research findings in particular areas and synthesise results. Such reviews would be carried out on an annual basis. This is a value-added process which puts information in a more convenient form to support decision making in professional practice.

- Posting of policies, standards, architectures, product evaluations etc. by practitioners for peer review: this would be done on a voluntary basis to facilitate knowledge sharing, development of "best practice" and industry standardisation. Clearly, commercial interests will be a limiting factor in such knowledge sharing, as many organisations will be protective of their intellectual property, especially in strategic areas.

- Listservers to promote discussion on particular issues: this would facilitate transfer of tacit knowledge in the form of ideas and experiences between practitioners and researchers.

The project will include implementation of the system and evaluation of its effectiveness using a sample of IS professionals (field experiment).

\section{Potential Benefits}

Implementation of such a system would:

- Help to connect researchers and practitioners together

- Improve the impact of research on practice

- $\quad$ Provide a comprehensive knowledge base to support decision making by IS practitioners 
- $\quad$ Reduce the time lag between the development of new research knowledge and its application in practice

- Help to eliminate use of practices that don't work

- Improve the professionalism of IS practice, by moving towards evidence-based rather than opinion and experience based practice

As in the previous case study, use of this system would also be beneficial to consumers. The IS profession lacks accountability because of the wide knowledge gulf between IS professionals and end users - practitioners often play on this by using technical jargon that end users do not understand, and thus avoid having their decisions scrutinized. Making the latest research evidence available to IS consumers would remove this knowledge gulf, and would help to improve accountability and the quality of IS practices.

\section{Potential Barriers}

There are a number of potential barriers to successful implementation of this system, due primarily to the differences between medical and IS practice:

- Scepticism of academic research: in general, IS practitioners are sceptical of the value of academic research, which would be a barrier to adoption and use of the system. However if the knowledge contained in the system is found to be useful, and a critical mass of practitioners start using the system, normative influences will take over (Green, 1998).

- Lack of evidence: in many areas of IS practice, there simply isn't a sufficient body of empirical research to conduct a systematic review. Because new technologies are being developed all the time, it is difficult for empirical research to keep pace with these advances.

- Strength of evidence: the conclusions of IS research studies tend to be much weaker than in medicine-few studies in the IS field would satisfy the requirements of medical "evidence". Because most IS interventions are applied at the organisational level, randomised clinical trials, which are the standard tool for obtaining evidence in medicine, are not applicable — in fact, many researchers have argued that experimental methods are inappropriate in the IS field. IS research relies heavily on qualitative research methods, which does not tend to result in definitive conclusions about the effectiveness of practices.

- Relevance of IS research: A number of authors have criticised IS research for not addressing questions that are relevant to practice and not producing knowledge that practitioners can readily use (Keen, 1991; Galliers, 1994; Benbasat and Zmud, 1999; Davenport and Markus, 1999). This is a "supply side" issue that is difficult to address. However if research results are disseminated in practice, this should improve the relevance of the research through natural feedback from practitioners to those conducting research. In addition, listservers provide a direct channel for practitioners to influence research priorities.

Gibbons et al (1994) argue that universities no longer have a monopoly on knowledge production, and that especially in high tech fields, many of the ideas, methods and technologies originate from practice than from academic research. This suggests that it will be important to take an inclusive approach in this project: rather than restricting knowledge sources to academic research, it is important to also include industry based knowledge sources.

\section{Conclusion}

For research to make a practical difference, research results must be readily available to practitioners, and must be actively used and implemented in everyday practice (Jordens et al, 1998; Phillips, 1998). For this reason, disciplines need a common information infrastructure that connects those who produce knowledge 
(researchers) to those who need to apply that knowledge (practitioners). The World Wide Web provides such an infrastructure for linking research and professional practice. This paper has described a system which makes the latest medical research available on-line to support clinical decision making at the point of care. It also describes a project currently in progress to develop a similar system to support IS practice. Neither of these projects would have been possible prior to the development of the World Wide Web, and illustrate how it has the potential to improve practices, and also to allow consumers to become more informed.

\section{A Paradigm for Evidence Based Practice?}

The systems movement was founded with the objective to "investigate the similarities of concepts, laws and models from various fields and to help in useful transfers from one field to another" (von Bertalanffy, 1968). Systems theory uses the process of generalisation as an approach to achieving such transfers. A problem in a particular domain can be generalised to a systems problem by removing all domain specific aspects (Klir, 1985). The solution to the systems problem can then be applied in a wide range of domains.

Evidence based medicine (EBM) is an approach for improving the effectiveness of medical practice. More generally however, it provides a conceptual framework for building more effective links between research and practice. The problems addressed by EBM are experienced to a greater or lesser extent in all applied disciplines, and the methods used (e.g. systematic reviews) could be applied in any field of research. It simply reflects the maturity of medicine as a profession in developing a solution to this problem first. Similar principles could be used in any applied discipline to reduce the time lag between the production of new research knowledge and its application in practice. The principles of EBM can be generalised to the systems level to develop a general paradigm for evidence based practice, which can then be applied in multiple domains.

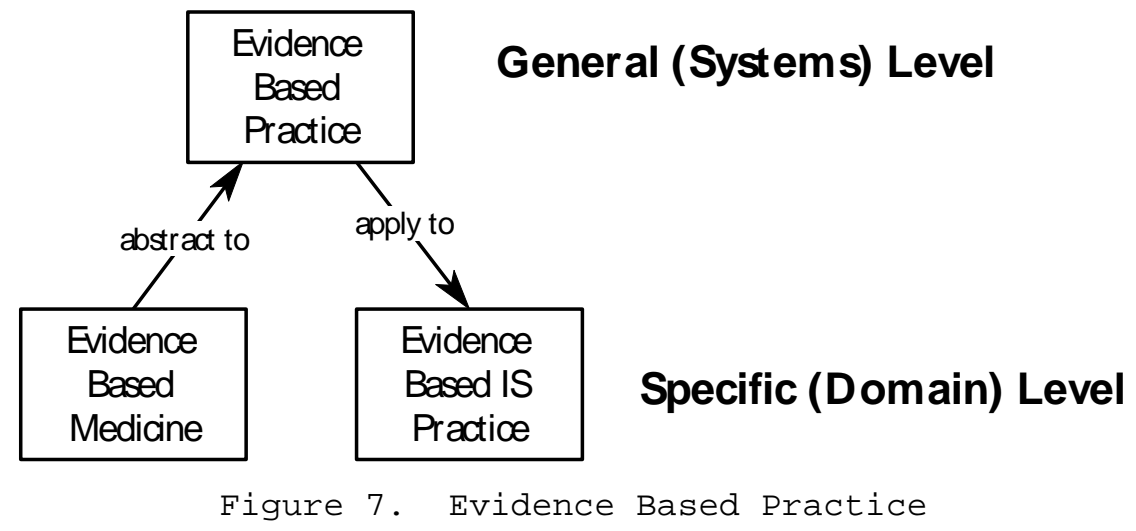

The key elements of evidence based practice, as embodied in the case studies described, are:

- Systematic reviews: synthesis of research literature to support decision making in practice.

- On-line literature searching tools: while these are standard "tools of the trade" for researchers, few practitioners have access to them in the workplace.

- Posting of practitioner-developed "knowledge products" (e.g. policies, standards, procedures: sharing of explicit knowledge to support development of best practice and industry standardisation.

- Listservers: informal channels for sharing knowledge between researchers and practitioners in a particular area of interest. 
Using the Web to Connect Research and Practice

\section{Inter-Organisational Knowledge Management}

The field of knowledge management is relatively new, and so far there have been few detailed case studies of knowledge management projects in practice (Moody and Shanks, 2001). Almost all previous empirical studies of knowledge management projects have taken place within a single organisation. Prior to the emergence of the Internet, information systems (and therefore most knowledge management initiatives) were constrained within the scope of a single organisation for technical reasons.

The Internet provides the infrastructure for connecting the producers of research knowledge (researchers) with the intended consumers of this knowledge (practitioners and consumers). The projects described in this paper illustrate how using the Web, knowledge management principles can be applied across a whole industry or profession. This leads to an expanded notion of knowledge management from the level of individual organisations to an entire discipline. This suggests a need for further research into interorganisational knowledge management.

The first case study also illustrates how such a knowledge management exercise could be extended to consumers as well as practitioners. As knowledge is power, this erodes the power base of practitioners, which they are likely to resist, but ultimately it is good for society. Intelligent consumers will help to improve professional standards and increase accountability.

\section{References}

AYRES, D.H.M. and CLINTON, S. (1997): "The User Connection: Making the Clinical Information Systems Vision Work in NSW Health", Health Informatics Conference (HIC '97), Sydney, Australia.

BADDELEY, A.D. (1994): “The Magical Number Seven: Still Magic After All These Years?”, Psychological Review, 101, 2 ,

BADDELEY, A.D. (1999): Essentials of Human Memory, Psychology Press, Hove [England].

BENBASAT, I. and ZMUD, R.W. (1999): "Empirical Research in Information Systems: The Practice of Relevance", MIS Quarterly, 23, 1, March.

CHALMERS, I. (1993): "The Cochrane Collaboration: Preparing, Maintaining and Disseminating Systematic Reviews of the Effects of Health Care”. Doing More Good Than Harm: The Evaluation of Health Care Interventions, K.S. Warren and F. Mosteller, (Eds.), Annals of the New York Academy of Sciences.

COCHRANE, A.L. (1972): Effectiveness and Efficiency: Random Reflections on Health Services, Royal Society of Medicine Press, London.

DAVENPORT, T.H., DE LONG, D.W. and BEERS, M.C. (1998): "Successful Knowledge Management Projects", Sloan Management Review, 39, 2, pp. 43-52,

DAVENPORT, T.H. and MARKUS, M.L. (1999): "Rigour vs Relevance Revisited: Response to Benbasat and Zmud", MIS Quarterly, 23, 1, March.

DAVENPORT, T.H. and PRUSAK, L. (1998): Working Knowledge: How Organisations Manage What They Know, Harvard Business School Press, Boston, Massachusetts.

EYSENCK, M.W. and KEANE, M.T. (2000): Cognitive Psychology: A Student's Handbook, Lawrence Erlbaum, Hove.

GALLIERS, R.D. (1994): "Relevance and Rigour in Information Systems Research: Some Personal Reflections on Issues Facing the Information Systems Research Community", Proceedings of the IFIP TC8 Conference on Business Process Reengineering: Information Systems and Challenges, Gold Coast, Australia.

GIBBONS, M., LIMOGES, C., NOWOTNY, H., SCHWARTZMAN, S., SCOTT, P. and TROW, M. (1994): The New Production of Knowledge: The Dynamics of Science and Research in Contemporary Societies, Sage Publications.

GOODWIN, P. (2000): “Improving the voluntary integration of statistical forecasts and judgement”, International Journal of Forecasting, 16, pp. 85-99,

GREEN, C. (1998): "Normative Influence on the Acceptance of Information Technology", Small Group Research, 29, 1, February. 
HANSEN, M., NOHRIA, H. and TIERNEY, T. (1999): “What's Your Strategy For Managing Knowledge?”, Harvard Business Review, March/April.

HARDGRAVE, B.C. and WALSTROM, K.A. (1997): "Forums for MIS Scholars", Communications of the ACM, 40, 11, November.

JORDENS, C.F.C., HAWE, P., IRWIG, L.M., HENDERSON-SMART, D.J., RYAN, M., DONOGHUE, D.A., GABB, R.G. and FRASER, I.S. (1998): "Use of Systematic Review of Randomised Trials by Australian Neonatologists and Obstetricians", Medical Journal of Australia, March 16.

KEEN, P.G.W. (1991): "Relevance and Rigour in Information Systems Research: Improving Quality, Confidence, Cohesion and Impact", Information Systems Research: Contemporary Approaches and Emergent Traditions, H.-E. Nissen, H.K. Klein and R. Hirschheim, (Eds.), Elsevier Science Publishers (North Holland).

KIDD, M.R. and VEALE, B.M. (1998): "How safe is Australian general practice and how can it be made safer?", Medical Journal of Australia, July 20.

KLIR, G.J. (1985): Architecture of Systems Problem Solving, Plenum Press, New York.

LAWRENCE, M.J. and SIM, W. (1999): "Prototyping a financial DSS”, Omega, 27, 4, pp. 445-450,

LEAPE, L. (1994): "Error in Medicine", Journal of the American Medical Association (JAMA), 272,

LIM, J. and O'CONNOR, M. (1995): "Judgemental adjustment of initial forecasts: its effectiveness and biases", Journal of Behavioural Decision Making, 8, pp. 149-168,

MILLER, G.A. (1956): “The Magical Number Seven, Plus Or Minus Two: Some Limits On Our Capacity For Processing Information", The Psychological Review, March.

MILNE, E.B.G.W.A. (2002): Drugs: Synonyms \& Properties

Second Edition, Ashgate Publishing Company, Burlington, Vermont, USA.

MOODY, D.L. (2000): "Building Links Between IS Research and Professional Practice: Improving the Relevance and Impact of IS Research", International Conference on Information Systems (ICIS'O0), R.A. Weber and B. Glasson, (Eds.), Brisbane, Australia.

MOODY, D.L. and SHANKS, G.G. (2001): "Using On-Line Medical Knowledge to Support Evidence Based Practice: A Case Study of a Successful Knowledge Management Project". Knowledge Media in Healthcare: Opportunities and Challenges, R. Grütter, (Ed.), Idea Group Publishing.

NONAKA, I. and TAKEUCHI, H. (1995): The Knowledge Creating Company, Oxford University Press, New York.

PHILLIPS, P.A. (1998): "Disseminating and Applying the Best Evidence”, Medical Journal of Australia, March.

SACKETT, D.L., RICHARDSON, W.S., ROSENBERG, W. and HAYNES, R.B. (1997): Evidence Based Medicine: How to Practice and Teach EBM, Churchill Livingstone, New York.

STANDISH GROUP (1995): The CHAOS Report into Project Failure, The Standish Group International Inc. Available on-line at http://www.standishgroup.com/visitor/chaos.htm.

SVEIBY, K.-E. (1997): The New Organisational Wealth: Managing and Measuring Knowledge-Based Assets, Berret-Koehler Publishers, San Francisco.

TURBAN, E. (1995): Decision support and expert systems: management support systems, (4th ed.), Prentice Hall Inc., Engelwood Cliffs, N.J. USA.

VON BERTALANFFY, L. (1968): General Systems Theory: Foundations, Development, Applications, Braziller.

WEBER, R.A. (1997): Ontological Foundations Of Information Systems, Coopers And Lybrand Accounting Research Methodology Monograph No. 4, Coopers And Lybrand, Melbourne, Australia.

WEED, L.L. (1997): "New Connections Between Medical Knowledge and Patient Care”, British Medical Journal, 315, July 26.

WILSON, R.M., HARRISON, B.T., GIBBERD, R.W. and HAMILTON, J.D. (1999): "An Analysis of the Causes of Adverse Events From the Quality in Australian Health Care Study", The Medical Journal of Australia, May 3.

WILSON, R.M., RUNCIMAN, W.B., GIBBERD, R.W., HARRISON, B.T., NEWBY, L. and HAMILTON, J.D. (1995): "The Quality in Australian Health Care Study", The Medical Journal of Australia, November 6. 